\title{
Impact of CP phases on SUSY particle production and decays
}

\author{
Alfred Bartl* \\ Institut für Theoretische Physik, Universität Wien, A-1090 Vienna \\ E-mail: bartl@ap.univie.ac.ati
}

\section{Hans Fraas}

Institut für Theoretische Physik, Universität Würzburg, D-97074 Würzburg

\section{Stefan Hesselbach}

Institut für Theoretische Physik, Universität Wien, A-1090 Vienna

\section{Keisyo Hidaka}

Department of Physics, Tokyo Gakugei University, Koganei, Tokyo 184-8501, Japan

\section{Thomas Kernreiter}

Institut für Theoretische Physik, Universität Wien, A-1090 Vienna

\section{Olaf Kittel}

Institut für Theoretische Physik, Universität Würzburg, D-97074 Würzburg

\section{Werner Porod}

Institut für Theoretische Physik, Universität Zürich, CH-8057 Zürich

ABstRACT: We report on the results of a phenomenological study of top squarks $\left(\tilde{t}_{1,2}\right)$ and bottom squarks $\left(\tilde{b}_{1,2}\right)$ in the Minimal Supersymmetric Standard Model (MSSM) with complex parameters $A_{t}, A_{b}, \mu$ and $M_{1}$. In particular we focus on the CP phase dependence of the branching ratios of $\tilde{t}_{1,2}$ and $\tilde{b}_{1,2}$ decays. We find that the effect of the phases on the $\tilde{t}_{1,2}$ and $\tilde{b}_{1,2}$ decays can be quite significant in a large region of the MSSM parameter space. We also study a CP asymmetry in sfermion decays.

\section{Introduction}

Supersymmetry (SUSY) is one of the best studied extensions [i] of the Standard Model (SM). SUSY gives us also the benefit of introducing potential new sources of CP violation ${ }^{*}$ Speaker. 
[2]. As the small amount of CP violation in the SM is not sufficient to explain the baryon asymmetry of the universe, it is necessary to study all implications of the complex SUSY parameters. The search for SUSY will be one of the main goals of all future colliders. An $e^{+} e^{-}$linear collider will be an ideal machine for the determination of the underlying SUSY parameters [ij]

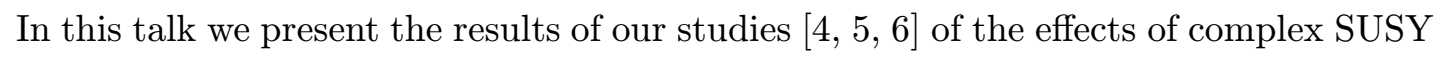
parameters on the phenomenology of the scalar top quark and scalar bottom quark system. Analysing the properties of 3rd generation sfermions is particularly interesting, because of the effects of the large Yukawa couplings. The lighter sfermion mass eigenstates may be among the light SUSY particles and they could be investigated at $e^{+} e^{-}$linear colliders

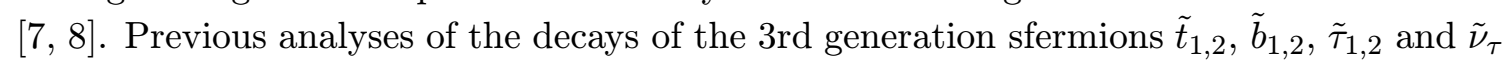
in the Minimal Supersymmetric Standard Model (MSSM) with real parameters have been

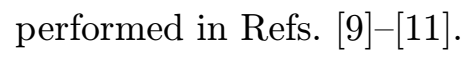

In the MSSM the SUSY parameters $A_{f}, \mu$ and $M_{i}(i=1,2,3)$ are in general complex, where $A_{f}$ is the trilinear scalar coupling parameter of the sfermion $\tilde{f}_{i}, \mu$ is the Higgshiggsino mass parameter and $M_{1}, M_{2}$ and $M_{3}$ are the $\mathrm{U}(1), \mathrm{SU}(2)$ and $\mathrm{SU}(3)$ gaugino mass parameters, respectively. We will first study the phase dependence of the decay branching ratios of the top squarks and bottom squarks, which are CP-even observables. As we will show, the phase dependence of these decay branching is indeed suitable to obtain informations about the SUSY CP phases. The situation is quite similar to that of the third generation slepton system, where the decay branching ratios of the staus $\tilde{\tau}_{1,2}$ and $\tau$-sneutrino $\tilde{\nu}_{\tau}$ can be used to get information on the phases of the stau and gauginohiggsino sectors [i1 $\overline{2}$ i]. In our study we will use the MSSM as a general framework and assume that the parameters $A_{t}, A_{b}, \mu$ and $M_{1}$ have the phases $\varphi_{A_{t}}, \varphi_{A_{b}}, \varphi_{\mu}$ and $\varphi_{\mathrm{U}(1)}$, respectively (taking $M_{2,3}$ real). We take into account explicit CP violation in the Higgs sector [1] parameters which follow from the experimental data on the rare decay $b \rightarrow s \gamma$ [i $\left[\bar{b}_{i}\right]$.

We will also consider a CP-odd observable in sfermion decays, which provides a more direct signal for the presence of $\mathrm{CP}$ phases. This observable is a CP-sensitive asymmetry

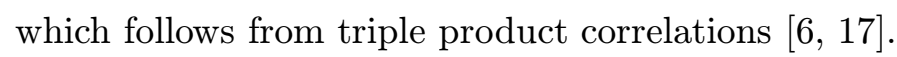

\section{Decay Branching Ratios of Top Squarks and Bottom Squarks}

Considering first top squark and bottom squark production, the reaction $e^{+} e^{-} \rightarrow \tilde{q}_{i} \overline{\tilde{q}}_{j}$, $\tilde{q}_{i}=\tilde{t}_{i}, \tilde{b}_{i}$, proceeds via $\gamma$ and $Z$ exchange in the $s$-channel. The tree-level cross sections $\left[\tilde{i}_{i}\right]$ of these reactions do not explicitly depend on the phases $\varphi_{\mu}$ and $\varphi_{A_{q}}$, because the $Z \tilde{q}_{i} \tilde{q}_{i}$ couplings are real and in $e^{+} e^{-} \rightarrow \tilde{q}_{1} \overline{\tilde{q}}_{2}$ only $Z$ exchange contributes. The cross sections depend only on the mass eigenvalues $m_{\tilde{q}_{1,2}}$ and on the mixing angles $\cos ^{2} \theta_{\tilde{q}}$. Therefore, they depend only implicitly on the phases via the $\cos \left(\varphi_{\mu}+\varphi_{A_{q}}\right)$ dependence of $m_{\tilde{q}_{1,2}}$ and $\theta_{\tilde{q}}$.

In the following we will present numerical results for the phase dependences of the $\tilde{t}_{i}$ and $\tilde{b}_{i}$ partial decay widths and branching ratios. We will treat the fermionic decays

$$
\tilde{q}_{i} \rightarrow q^{\prime}+\tilde{\chi}_{k}^{ \pm}, i=1,2, k=1,2
$$


and

$$
\tilde{q}_{i} \rightarrow q+\tilde{\chi}_{k}^{0}, i=1,2, k=1, . ., 4
$$

and the bosonic decays

$$
\begin{gathered}
\tilde{q}_{i} \rightarrow W^{ \pm}+\tilde{q}_{j}^{\prime}, i=1,2, j=1,2, j \leq i \\
\tilde{q}_{i} \rightarrow H^{ \pm}+\tilde{q}_{j}^{\prime}, i=1,2, j=1,2, j \leq i \\
\tilde{q}_{2} \rightarrow Z+\tilde{q}_{1}
\end{gathered}
$$

and

$$
\tilde{q}_{2} \rightarrow H_{i}+\tilde{q}_{1}, i=1,2,3 .
$$

These partial decay widths depend on the SUSY parameters of the squark system $M_{\tilde{Q}}$, $M_{\tilde{U}}, M_{\tilde{D}}, \tan \beta,|\mu|, \varphi_{\mu},\left|A_{t}\right|, \varphi_{A_{t}},\left|A_{b}\right|, \varphi_{A_{b}}$, which determine the mass eigenvalues and mixing angles of the top and bottom squarks. In addition, in the chargino sector the $S U(2)$ gaugino mass parameter $M_{2}$ enters. The mass eigenvalues and mixing of the neutralino depend also on the $U(1)$ gaugino mass parameter $M_{1}$ with phase $\varphi_{\mathrm{U}(1)}$ which, therefore, influences the partial decay widths of the top and bottom squarks into neutralinos. The main parameter of the Higgs sector is the charged Higgs boson mass $m_{H^{ \pm}}$, in addition to the parameters already introduced.

We calculate the partial decay widths in Born approximation. In some cases the one-

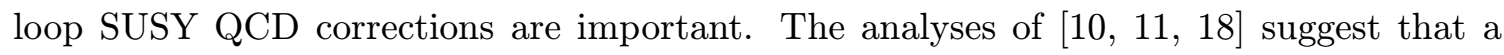
significant part of the one-loop SUSY QCD corrections to the partial widths of $\tilde{t}_{i}$ and $\tilde{b}_{i}$ decays (where the bottom Yukawa coupling is involved) can be incorporated by using an appropriately corrected bottom quark mass. In this spirit we calculate the tree-level widths of the $\tilde{t}_{i}$ and $\tilde{b}_{i}$ decays by using on-shell masses for the kinematic terms (such as phase space factors) and by taking running $t$ and $b$ quark masses for the Yukawa couplings. For definiteness we take $m_{t}^{\text {run }}\left(m_{Z}\right)=150 \mathrm{GeV}, m_{t}^{\text {on-shell }}=175 \mathrm{GeV}, m_{b}^{\text {run }}\left(m_{Z}\right)=3 \mathrm{GeV}$ and $m_{b}^{\text {on-shell }}=5 \mathrm{GeV}$. This approach leads to an "improved" Born approximation which takes into account an essential part of the one-loop SUSY QCD corrections to the $\tilde{t}_{i}$ and $\tilde{b}_{i}$ partial decay widths and predicts their phase dependences more accurately than the "naive" tree-level calculation. In the calculation of the $\mathrm{CP}$ violating effects in the neutral Higgs sector we take the program FeynHiggs-2.0.2 of [i]

In the numerical analysis we impose as theoretical constraint the approximate necessary condition for the tree-level vacuum stability [i] $\left.\underline{1}_{1}^{1}\right]$. Furthermore, as experimental constraints

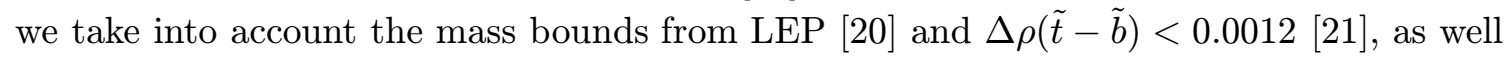
as $2.0 \times 10^{-4}<B(b \rightarrow s \gamma)<4.5 \times 10^{-4}[1 \overline{0} \overline{6}]$ ] assuming the Kobayashi-Maskawa mixing also for the squark sector. For the calculation of the $b \rightarrow s \gamma$ width we use the formula of

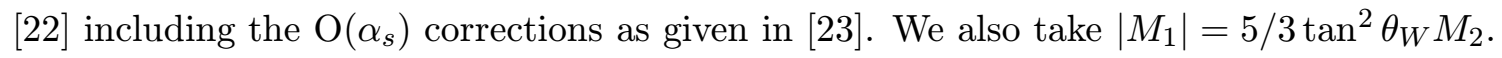

As a first example we show in Fig. ${ }_{1}^{1} \overline{1}_{1}^{\prime}(\mathrm{a})$ the contour plot for $B\left(\tilde{t}_{1} \rightarrow \tilde{\chi}_{1}^{0} t\right)$ as a function of $\varphi_{A_{t}}$ and $\varphi_{\mu}$ for $\left(m_{\tilde{t}_{1}}, m_{\tilde{t}_{2}}, m_{\tilde{b}_{1}}\right)=(350,700,170) \mathrm{GeV}, \tan \beta=6, M_{2}=300 \mathrm{GeV},|\mu|=$ $500 \mathrm{GeV},\left|A_{t}\right|=\left|A_{b}\right|=800 \mathrm{GeV}, \varphi_{\mathrm{U}(1)}=\varphi_{A_{b}}=0$ and $m_{H^{ \pm}}=600 \mathrm{GeV}$, assuming $M_{\tilde{Q}}>$ $M_{\tilde{U}}$. For the parameters chosen the $\varphi_{A_{t}}$ dependence is stronger than the $\varphi_{\mu}$ dependence. The reason is that these phase dependences are caused mainly by the $\tilde{t}_{L}-\tilde{t}_{R}$ mixing term, 

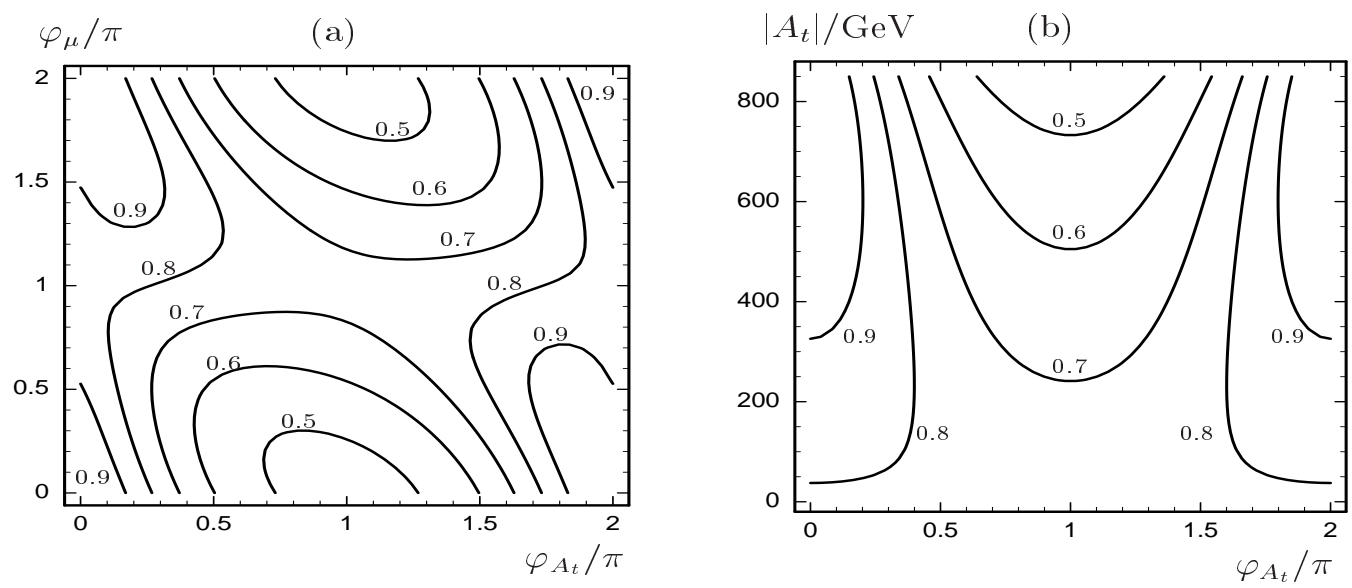

Figure 1: Contours of $B\left(\tilde{t}_{1} \rightarrow \tilde{\chi}_{1}^{0} t\right)$ for $\tan \beta=6, M_{2}=300 \mathrm{GeV},|\mu|=500 \mathrm{GeV}, \varphi_{\mathrm{U}(1)}=\varphi_{A_{b}}=0$, $m_{\tilde{t}_{1}}=350 \mathrm{GeV}, m_{\tilde{t}_{2}}=700 \mathrm{GeV}, m_{\tilde{b}_{1}}=170 \mathrm{GeV}, m_{H^{ \pm}}=600 \mathrm{GeV}$, with (a) $\left|A_{t}\right|=\left|A_{b}\right|=800 \mathrm{GeV}$ and (b) $\varphi_{\mu}=0,\left|A_{b}\right|=\left|A_{t}\right|$, assuming $M_{\tilde{Q}}>M_{\tilde{U}}$.

where the $\varphi_{\mu}$ dependence is suppressed by $\cot \beta$. The $\varphi_{\mu}$ dependence is somewhat more pronounced for $\varphi_{A_{t}} \approx \pi$ than for $\varphi_{A_{t}} \approx 0,2 \pi$. In Fig. 禀 (b) we show the contour plot of $B\left(\tilde{t}_{1} \rightarrow \tilde{\chi}_{1}^{0} t\right)$ as a function of $\varphi_{A_{t}}$ and $\left|A_{t}\right|$ for $\varphi_{\mu}=0$ and $\left|A_{t}\right|=\left|A_{b}\right|$. Clearly, the $\varphi_{A_{t}}$ dependence is stronger for larger values of $\left|A_{t}\right|$.

For the heavier top squark $\tilde{t}_{2}$ more decay channels are open. In Fig. $\overline{2}_{1}^{\prime}(\mathrm{a})$ we show the branching ratios for $\tilde{t}_{2} \rightarrow \tilde{\chi}_{1,2}^{+} b$ and $\tilde{t}_{2} \rightarrow \tilde{\chi}_{2,3,4}^{0} t$ as a function of $\varphi_{A_{t}}$ for $\left(m_{\tilde{t}_{1}}, m_{\tilde{t}_{2}}, m_{\tilde{b}_{1}}\right)=$ $(350,800,170) \mathrm{GeV}, \tan \beta=6, M_{2}=300 \mathrm{GeV},|\mu|=500 \mathrm{GeV},\left|A_{b}\right|=\left|A_{t}\right|=500 \mathrm{GeV}, \varphi_{\mu}=$ $\varphi_{\mathrm{U}(1)}=\varphi_{A_{b}}=0, m_{\tilde{t}_{1}}=350 \mathrm{GeV}, m_{\tilde{t}_{2}}=800 \mathrm{GeV}, m_{\tilde{b}_{1}}=170 \mathrm{GeV}$ and $m_{H^{ \pm}}=350 \mathrm{GeV}$, assuming $M_{\tilde{Q}}>M_{\tilde{U}}$. The $\varphi_{A_{t}}$ dependence of $B\left(\tilde{t}_{2} \rightarrow \tilde{\chi}_{1,2}^{+} b\right)$ is due to a direct phase effect, which explains that the shape of $B\left(\tilde{t}_{2} \rightarrow \tilde{\chi}_{1,2}^{+} b\right)$ is like $\left(1 \pm \cos \varphi_{A_{t}}\right)$. Also the phase dependence of the branching ratios into neutralinos is mainly due to a direct phase effect. $B\left(\tilde{t}_{2} \rightarrow \tilde{\chi}_{2}^{0} t\right)$ has a very weak phase dependence like $\left(10+\cos \varphi_{A_{t}}\right)$. In $\Gamma\left(\tilde{t}_{2} \rightarrow \tilde{\chi}_{3}^{0} t\right)$ the mixing phase enters, resulting in a shape like $\left(1+\cos \varphi_{A_{t}}\right)$ for the branching ratio. Similarly, $B\left(\tilde{t}_{2} \rightarrow \tilde{\chi}_{4}^{0} t\right)$ behaves like $\left(1-\cos \varphi_{A_{t}}\right)$.

In Fig. $\overline{2}$ (b) we show the branching ratios for the bosonic decays $\tilde{t}_{2} \rightarrow Z \tilde{t}_{1}$ and $\tilde{t}_{2} \rightarrow H_{i} \tilde{t}_{1}(i=1,2,3)$ for the same parameter values as above. The shape of $B\left(\tilde{t}_{2} \rightarrow Z \tilde{t}_{1}\right)$ is like $\left(1-\cos \varphi_{A_{t}}\right)$, which is caused by the $\theta_{\tilde{t}}$ dependence of the corresponding coupling. Quite generally, the phase dependence of $\Gamma\left(\tilde{t}_{2} \rightarrow H_{k} \tilde{t}_{1}\right)$ is the result of a complicated interplay among the phase dependences of the neutral Higgs boson masses, the top squark mixing matrix elements, the neutral Higgs mixing matrix elements and the direct top squark-Higgs couplings. In the present example the $\varphi_{A_{t}}$ dependence of the partial widths $\Gamma\left(\tilde{t}_{2} \rightarrow H_{1,2,3} \tilde{t}_{1}\right)$ is mainly due to the $\varphi_{A_{t}}$ dependence of the top squark mixing matrix and the squark-Higgs couplings, whereas the $\varphi_{A_{t}}$ dependence of the neutral Higgs mixing 
(a)

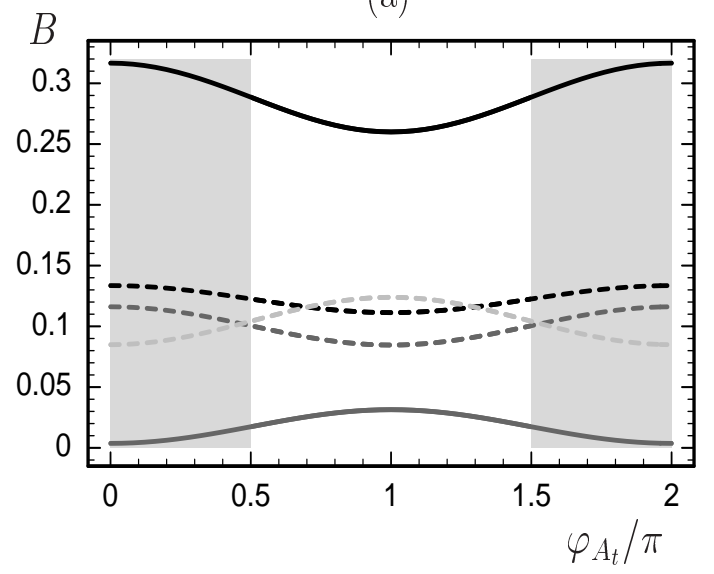

(b)

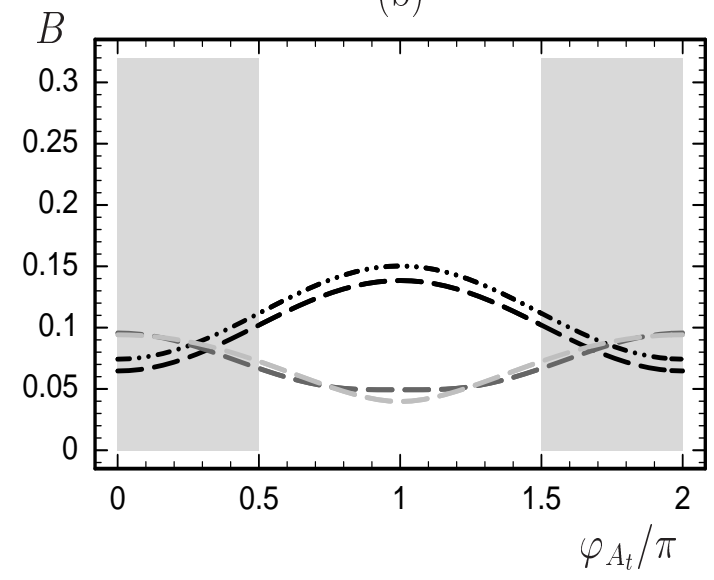

Figure 2: $\varphi_{A_{t}}$ dependence of branching ratios of the decays (a) $\tilde{t}_{2} \rightarrow \tilde{\chi}_{1 / 2}^{+} b$ (solid, black/gray), $\tilde{t}_{2} \rightarrow \tilde{\chi}_{2 / 3 / 4}^{0} t$ (dashed, black/gray/light gray) and (b) $\tilde{t}_{2} \rightarrow Z \tilde{t}_{1}$ (dashdotdotted), $\tilde{t}_{2} \rightarrow H_{1 / 2 / 3} \tilde{t}_{1}$ (long dashed, black/gray/light gray) for $\tan \beta=6, M_{2}=300 \mathrm{GeV},|\mu|=500 \mathrm{GeV},\left|A_{b}\right|=\left|A_{t}\right|=$ $500 \mathrm{GeV}, \varphi_{\mu}=\varphi_{\mathrm{U}(1)}=\varphi_{A_{b}}=0, m_{\tilde{t}_{1}}=350 \mathrm{GeV}, m_{\tilde{t}_{2}}=800 \mathrm{GeV}, m_{\tilde{b}_{1}}=170 \mathrm{GeV}$ and $m_{H^{ \pm}}=350 \mathrm{GeV}$, assuming $M_{\tilde{Q}}>M_{\tilde{U}}$. Only the decay modes with $B \gtrsim 1 \%$ are shown. The shaded areas mark the region excluded by the experimental limit $B(b \rightarrow s \gamma)<4.5 \times 10^{-4}$.

matrix is less pronounced in this case.

Coming now to the discussion of the decays of the bottom squarks $\tilde{b}_{1,2}$, we show in Fig. $\underline{\underline{3}}_{1}$ the partial decay widths and the branching ratios of $\tilde{b}_{1} \rightarrow \tilde{\chi}_{1,2}^{0} b, H^{-} \tilde{t}_{1}, W^{-} \tilde{t}_{1}$ as a function of $\varphi_{A_{b}}$ for $m_{\tilde{b}_{1}}=350 \mathrm{GeV}, m_{\tilde{b}_{2}}=700 \mathrm{GeV}, m_{\tilde{t}_{1}}=170 \mathrm{GeV}, \tan \beta=30, m_{H^{ \pm}}=150 \mathrm{GeV}$, $M_{2}=200 \mathrm{GeV},|\mu|=300 \mathrm{GeV},\left|A_{b}\right|=\left|A_{t}\right|=600 \mathrm{GeV}, \varphi_{\mu}=\pi$ and $\varphi_{A_{t}}=\varphi_{\mathrm{U}(1)}=0$, assuming $M_{\tilde{Q}}>M_{\tilde{D}}$. In the region $0.5 \pi<\varphi_{A_{b}}<1.5 \pi$ the decay $\tilde{b}_{1} \rightarrow H^{-} \tilde{t}_{1}$ dominates. The $\varphi_{A_{b}}$ dependence of $\Gamma\left(\tilde{b}_{1} \rightarrow H^{-} \tilde{t}_{1}\right)$ is due to the behaviour of the squark-Higgs coupling. The partial decay widths $\Gamma\left(\tilde{b}_{1} \rightarrow \tilde{\chi}_{1,2}^{0} b\right)$ are almost $\varphi_{A_{b}}$ independent because the $\varphi_{A_{b}}$ dependence of the bottom squark mixing matrix nearly vanishes for $\tan \beta=30$. Hence the $\varphi_{A_{b}}$ dependence of the branching ratios $B\left(\tilde{b}_{1} \rightarrow \tilde{\chi}_{\tilde{\sigma}_{2}}^{0} b\right)$ is caused by that of the total decay width. $\Gamma\left(\tilde{b}_{1} \rightarrow W^{-} \tilde{t}_{1}\right)$ is suppressed because $\tilde{b}_{1} \sim \tilde{b}_{R}$ and $\tilde{t}_{1} \sim \tilde{t}_{R}$ in this scenario.

We have also estimated what accuracy can be expected in the determination of the underlying MSSM parameters by a global fit of the observables (masses, branching ratios and production cross sections) measured typical linear collider experiments with polarized beams. We have found that under favourable conditions the fundamental MSSM parameters except $A_{t, b}$ can be determined with errors of $1 \%$ to $2 \%$, assuming an integrated luminosity of $1 \mathrm{ab}^{-1}$. The parameter $A_{t}$ can be determined within an error of $2-3 \%$ whereas the error of $A_{b}$ is likely to be of the order of $50 \%$. More details can be found in [4în. 

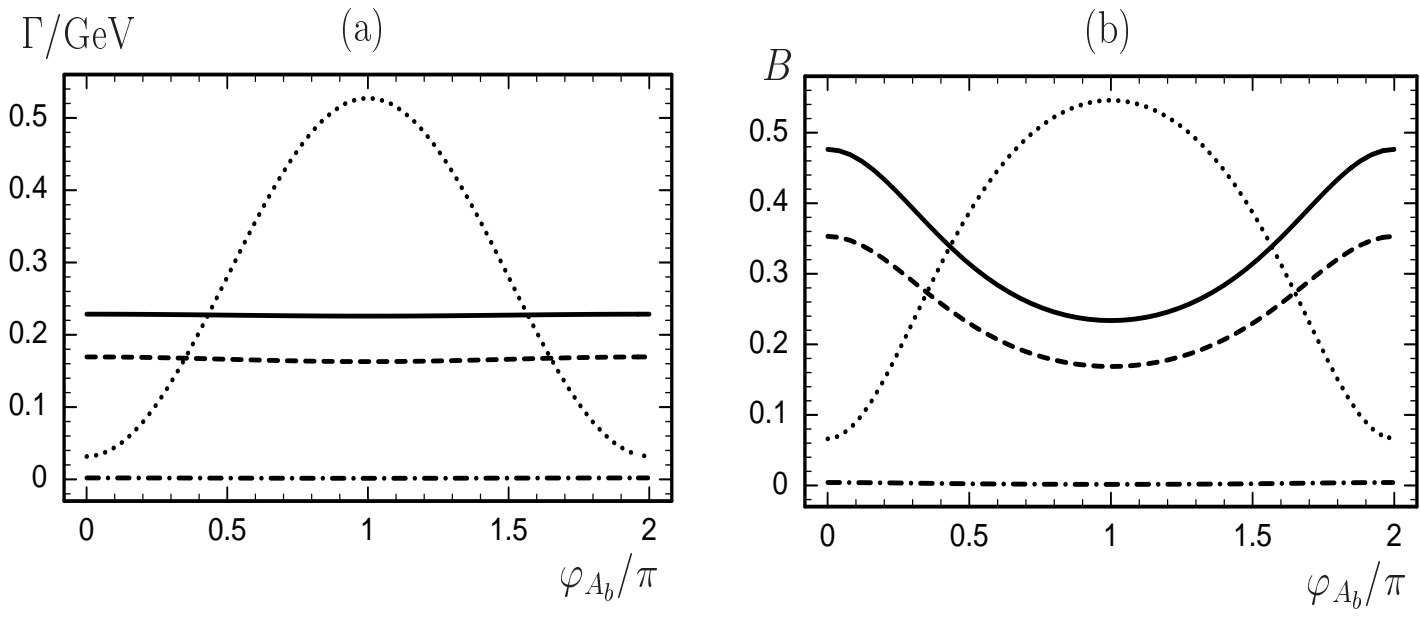

Figure 3: $\varphi_{A_{b}}$ dependences of (a) partial widths and (b) branching ratios of the decays $\tilde{b}_{1} \rightarrow \tilde{\chi}_{1}^{0} b$ (solid), $\tilde{b}_{1} \rightarrow \tilde{\chi}_{2}^{0} b$ (dashed), $\tilde{b}_{1} \rightarrow H^{-} \tilde{t}_{1}$ (dotted) and $\tilde{b}_{1} \rightarrow W^{-} \tilde{t}_{1}$ (dashdotted) for $\tan \beta=30$, $M_{2}=200 \mathrm{GeV},|\mu|=300 \mathrm{GeV},\left|A_{b}\right|=\left|A_{t}\right|=600 \mathrm{GeV}, \varphi_{\mu}=\pi, \varphi_{A_{t}}=\varphi_{\mathrm{U}(1)}=0, m_{\tilde{b}_{1}}=350 \mathrm{GeV}$, $m_{\tilde{b}_{2}}=700 \mathrm{GeV}, m_{\tilde{t}_{1}}=170 \mathrm{GeV}$ and $m_{H^{ \pm}}=150 \mathrm{GeV}$, assuming $M_{\tilde{Q}}>M_{\tilde{D}}$.

\section{CP Asymmetries in Sfermion Decays}

As the sfermions are scalar particles and they usually have two-body decay modes, it is not straightforward to construct a CP sensitive asymmetry involving their decays. In the case of top squarks one can use a three-body decay [1 $\overline{1}_{2} \overline{7}_{1}$, for the other sfermions one has to consider cascade decays. In our paper [6]'] we have considered the decay chain

$$
\tilde{f} \rightarrow f \tilde{\chi}_{j}^{0} \rightarrow f \tilde{\chi}_{1}^{0} Z \rightarrow f \tilde{\chi}_{1}^{0} \ell \bar{\ell}\left(f \tilde{\chi}_{1}^{0} q \bar{q}\right),
$$

where $\ell=e, \mu, \tau$, and $q$ denotes a quark. We have defined a T-odd correlation for the leptonic decay

$$
O_{\text {odd }}^{\ell}=\mathbf{p}_{f} \cdot\left(\mathbf{p}_{\ell} \times \mathbf{p}_{\bar{\ell}}\right),
$$

and for the hadronic decays as

$$
O_{\text {odd }}^{q}=\mathbf{p}_{f} \cdot\left(\mathbf{p}_{q} \times \mathbf{p}_{\bar{q}}\right),
$$

where $\mathbf{p}$ denotes the three-momentum of the corresponding fermion. We define the corresponding T-odd asymmetries as

$$
\mathcal{A}_{\mathrm{T}}^{\ell, q}=\frac{\Gamma\left(O_{\text {odd }}^{\ell, q}>0\right)-\Gamma\left(O_{\text {odd }}^{\ell, q}<0\right)}{\Gamma\left(O_{\text {odd }}^{\ell, q}>0\right)+\Gamma\left(O_{\text {odd }}^{\ell, q}<0\right)}=
$$

which by $C P T$ are also CP asymmetries. This CP asymmetry is similar to that proposed

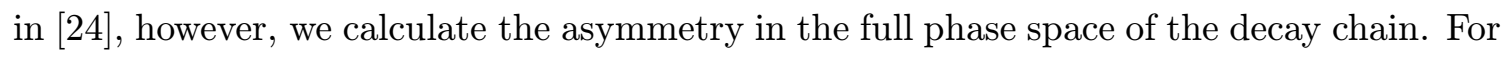



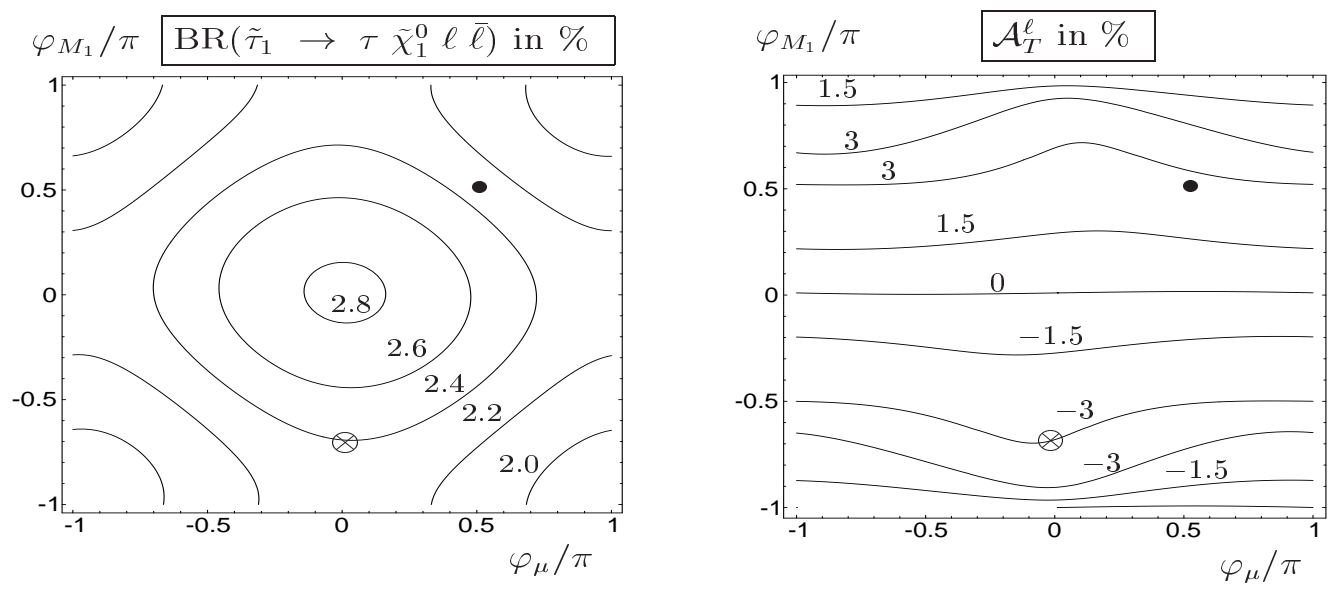

Figure 4: Contour lines of the branching ratio for $\tilde{\tau}_{1} \rightarrow \tilde{\chi}_{1}^{0} \tau \ell \bar{\ell}$ and asymmetry $A_{T}^{\ell}$ in the $\varphi_{M_{1}}-\varphi_{\mu}$ plane for $|\mu|=300 \mathrm{GeV}$ and $M_{2}=280 \mathrm{GeV}$, taking $\tan \beta=10, A_{\tau}=1000 \mathrm{GeV}, m_{\tilde{\tau}_{1}}=300 \mathrm{GeV}$, $m_{\tilde{\tau}_{2}}=800 \mathrm{GeV}$ for $M_{\tilde{E}}>M_{\tilde{L}}$. The point denoted by $\bullet$ is for the theoretical estimate of the necessary number of produced $\tilde{\tau}_{1}$ 's (see text).

the measurement of $\mathcal{A}_{\mathrm{T}}^{\ell}$ or $\mathcal{A}_{\mathrm{T}}^{q}$ it is necessary to be able to distinguish between the charges of $\ell^{+}$and $\ell^{-}$or $q$ and $\bar{q}$. In the case $\ell=e, \mu, \tau$ this should be possible experimentally on an event by event basis at an $e^{+} e^{-}$linear collider $\left[3 \overline{3}^{-1}\right.$. $\mathcal{A}_{\mathrm{T}}^{q}$ will be measureable in the case of $q=c, b$, where flavour reconstruction is possible [20 $2 \overline{5}]$.

As an example we have calculated $\mathcal{A}_{\mathrm{T}}^{\ell}$ for $\tilde{\tau}_{1}$ decay, considering the decay chain $\tilde{\tau}_{1} \rightarrow$ $\tau \tilde{\chi}_{2}^{0}, \tilde{\chi}_{2}^{0} \rightarrow Z \tilde{\chi}_{1}^{0}, Z \rightarrow \ell \bar{\ell}$, for $\ell=e, \mu, \tau$. As input parameters we have chosen $m_{\tilde{\tau}_{1}}=$ $300 \mathrm{GeV}, m_{\tilde{\tau}_{2}}=800 \mathrm{GeV}, \tan \beta=10,\left|A_{\tau}\right|=1000 \mathrm{GeV}, \varphi_{A_{\tau}}=0, m_{A}=800 \mathrm{GeV}$, $M_{2}=280 \mathrm{GeV}$, using the GUT relation $\left|M_{1}\right|=5 / 3 \tan ^{2} \Theta_{W} M_{2}$

In Fig. 'ita we show the contour lines for the branching ratio $\operatorname{BR}\left(\tau_{1} \rightarrow \tau \tilde{\chi}_{1}^{0} \ell \bar{\ell}\right)=$ $\operatorname{BR}\left(\tilde{\tau}_{1} \rightarrow \tau \tilde{\chi}_{2}^{0}\right) \times \operatorname{BR}\left(\tilde{\chi}_{2}^{0} \rightarrow Z \tilde{\chi}_{1}^{0}\right) \times \operatorname{BR}(Z \rightarrow \ell \bar{\ell})$ in the $\varphi_{M_{1}}-\varphi_{\mu}$ plane for $M_{2}=280 \mathrm{GeV}$ and $|\mu|=300 \mathrm{GeV}$. For $\operatorname{BR}\left(\tau_{1} \rightarrow \tau \tilde{\chi}_{1}^{0} \ell \bar{\ell}\right)$ we always sum over $\ell=e, \mu, \tau$. We choose $M_{\tilde{E}}>M_{\tilde{L}}$ since in this case the $\tilde{\tau}_{1}-\tau-\tilde{\chi}_{2}^{0}$ coupling $\left|a_{12}^{\tilde{\tau}}\right|$ is larger, which implies a larger branching ratio $\operatorname{BR}\left(\tilde{\tau}_{1} \rightarrow \tau \tilde{\chi}_{2}^{0}\right)$ than for $M_{\tilde{E}}<M_{\tilde{L}}$. In a large region of the parameter space we have $\operatorname{BR}\left(\tilde{\chi}_{2}^{0} \rightarrow Z \tilde{\chi}_{1}^{0}\right)=1$, and we take $\operatorname{BR}(Z \rightarrow \ell \bar{\ell})=0.1$. In Fig. 䒛 we show the $\varphi_{M_{1}}$ and $\varphi_{\mu}$ dependence of $\mathcal{A}_{\mathrm{T}}^{\ell}$. The value of $\mathcal{A}_{\mathrm{T}}^{\ell}$ depends stronger on $\varphi_{M_{1}}$, than on $\varphi_{\mu}$. The sign of $\mathcal{A}_{\mathrm{T}}^{\ell}$ is essentially determined by the sign of $\varphi_{M_{1}}$

The asymmetries $\mathcal{A}_{\mathrm{T}}^{b(c)}$ can be calculated from $\mathcal{A}_{\mathrm{T}}^{\ell}$ by replacing the leptonic $Z$ coupling by the $Z q \bar{q}$ coupling. This gives

$$
\mathcal{A}_{\mathrm{T}}^{b(c)} \approx 6.3(4.5) \times \mathcal{A}_{\mathrm{T}}^{\ell}
$$


Based on our results for the asymmetry $\mathcal{A}_{\mathrm{T}}^{\ell}$ in $\tilde{\tau}_{1} \rightarrow \tau \tilde{\chi}_{2}^{0} \rightarrow \tilde{\chi}_{1}^{0} \tau \ell^{+} \ell^{-}$and the branching ratio we give a theoretical estimate of the number of produced $\tilde{\tau}_{1}$ 's necessary to observe the T-odd asymmetry. As an example we take the point denoted by $\bullet$ in Fig. 商, with $\varphi_{\mu}=\pi / 2$ and $\varphi_{M_{1}}=\pi / 2$. For this point $\mathrm{BR} \approx 2.5 \times 10^{-2}$ and $\left|\mathcal{A}_{\mathrm{T}}^{\ell}\right| \approx 3 \times 10^{-2}$. For the decay $\tilde{\tau}_{1} \rightarrow b \bar{b} \tilde{\chi}_{1}^{0} \tau$, on the other hand, $\mathrm{BR} \approx 3.6 \times 10^{-2}$ and $\left|\mathcal{A}_{\mathrm{T}}^{b}\right| \approx 1.9 \times 10^{-1}$. In this example the asymmeties $\mathcal{A}_{\mathrm{T}}^{\ell, q}$ should be measurable at an $e^{+} e^{-}$linear collider with $\sqrt{s}=800 \mathrm{GeV}$ and an integrated luminosity of $500 \mathrm{fb}^{-1}$ for $m_{\tilde{\tau}_{1}}=300 \mathrm{GeV}$. It is clear that detailed Monte Carlo studies taking into account background and detector simulations are necessary to get a more precise prediction of the expected accuracy. For a Monte Carlo study on a T-odd observable in neutralino production and decay see [i2 $\left.\underline{2}_{\overline{6}}\right]$.

\section{Acknowledgements}

We thank J. W. F. Valle and the organizers of AHEP03 for creating an inspiring atmosphere at this conference. This work is supported by the 'Fonds zur Förderung der wissenschaftlichen Forschung' of Austria, FWF Projects No. P13139-PHY and No. P16592N02 and by the European Community's Human Potential Programme under contract HPRN-CT-2000-00149. W.P. has been supported by the Erwin Schrödinger fellowship No. J2272 of the 'Fonds zur Förderung der wissenschaftlichen Forschung' of Austria and partly by the Swiss 'Nationalfonds'.

\section{References}

[1] For reviews, see:

H. P. Nilles, Phys. Rep. 110 (1984) 1;

H. E. Haber and G. L. Kane, Phys. Rep. 117 (1985) 75;

R. Barbieri, Riv. Nuovo Cim. 11 (1988) 1.

[2] M. Dugan, B. Grinstein and L. J. Hall, Nucl. Phys. B 255 (1985) 413. A. Masiero and O. Vives, New J. Phys. 4 (2002) 4.

[3] J. A. Aguilar-Saavedra et al. [ECFA/DESY LC Physics Working GroupCollaboration], E. Accomando et al., Phys. Rep. 229 (1998) 1;

H. Murayama and M. E. Peskin, Ann. Rev. Nucl. Part. Sci. 46, (1996) 533

[arXiv:hep-ex/9606003];

J. L. Feng and M. M. Nojiri, arXiv:hep-ph/0210390;

H. U. Martyn and G. A. Blair, in Proceedings of the 4th International Workshop on Linear Colliders (LCWS 99), Sitges, Barcelona, Spain, 28 Apr - 5 May 1999, arXiv:hep-ph/9910416.

[4] A. Bartl, S. Hesselbach, K. Hidaka, T. Kernreiter, W. Porod, arXiv:hep-ph/0311338.

[5] A. Bartl, S. Hesselbach, K. Hidaka, T. Kernreiter, W. Porod, LC-TH-2003-041, arXiv:hep:ph/0306281; Phys. Lett. B 573 (2003) 153.

[6] A. Bartl, H. Fraas, T. Kernreiter and O. Kittel, arXiv:hep-ph/0306304.

[7] M. M. Nojiri, Phys. Rev. D 51 (1995) 6281 [arXiv:hep-ph/9412374];

M. M. Nojiri, K. Fujii and T. Tsukamoto, Phys. Rev. D 54 (1996) 6756 [arXiv:hep-ph/9606370]; 
E. Boos, H. U. Martyn, G. Moortgat-Pick, M. Sachwitz, A. Sherstnev and P. M. Zerwas, Eur. Phys. J. C 30 (2003) 395 [arXiv:hep-ph/0303110]

E. Boos, G. Moortgat-Pick, H. U. Martyn, M. Sachwitz and A. Vologdin, arXiv:hep-ph/0211040.

[8] A. Bartl, H. Eberl, S. Kraml, W. Majerotto, W. Porod and A. Sopczak, Z. Phys. C 76 (1997) 549 [arXiv:hep-ph/9701336];

A. Bartl, H. Eberl, S. Kraml, W. Majerotto and W. Porod, Eur. Phys. J. direct C 2 (2000) 6 [arXiv:hep-ph/0002115];

R. Keranen, A. Sopczak, H. Nowak and M. Berggren, Eur. Phys. J. direct C 7 (2000) 1;

A. Bartl, W. Majerotto and W. Porod, Z. Phys. C 64 (1994) 499 [Erratum-ibid. C 68 (1995) 518].

[9] A. Bartl, H. Eberl, K. Hidaka, S. Kraml, T. Kon, W. Majerotto, W. Porod, Y. Yamada, Phys. Lett. B 435 (1998) 118 [arXiv:hep-ph/9804265]; Phys. Lett. B 460 (1999) 157 [arXiv:hep-ph/9904417].

[10] K. Hidaka and A. Bartl, Phys. Lett. B 501 (2001) 78 [arXiv:hep-ph/0012021].

[11] H. Eberl, K. Hidaka, S. Kraml, W. Majerotto, Y. Yamada, Phys. Rev. D 62 (2000) 055006 [arXiv:hep-ph/9912463];

C. Weber, H. Eberl and W. Majerotto, Phys. Lett. B 572 (2003) 56; [arXiv:hep-ph/0308146].

[12] A. Bartl, K. Hidaka, T. Kernreiter and W. Porod, Phys. Lett. B 538 (2002) 137

[arXiv:hep-ph/0204071]; Phys. Rev. D 66 (2002) 115009 [arXiv:hep-ph/0207186].

[13] D. A. Demir, Phys. Rev. D 60 (1999) 055006 [arXiv:hep-ph/9901389]

M. Carena, J. R. Ellis, A. Pilaftsis and C. E. Wagner, Phys. Lett. B 495 (2000) 155 [arXiv:hep-ph/0009212];

M. Carena, J. R. Ellis, A. Pilaftsis and C. E. Wagner, Nucl. Phys. B 586 (2000) 92 [arXiv:hep-ph/0003180].

[14] A. Pilaftsis, Phys. Lett. B 435 (1998) 88 [arXiv:hep-ph/9805373];

A. Pilaftsis and C. E. Wagner, Nucl. Phys. B 553 (1999) 3 [arXiv:hep-ph/9902371];

S. Y. Choi, M. Drees and J. S. Lee, Phys. Lett. B 481 (2000) 57 [arXiv:hep-ph/0002287];

G. L. Kane and L. T. Wang, Phys. Lett. B 488 (2000) 383 [arXiv:hep-ph/0003198];

M. Carena, J. R. Ellis, A. Pilaftsis and C. E. Wagner, Nucl. Phys. B 625 (2002) 345 [arXiv:hep-ph/0111245];

M. Carena, J. R. Ellis, S. Mrenna, A. Pilaftsis and C. E. Wagner, Nucl. Phys. B 659 (2003) 145 [arXiv:hep-ph/0211467].

[15] S. Heinemeyer, Eur. Phys. J. C 22 (2001) 521 [arXiv:hep-ph/0108059];

M. Frank, S. Heinemeyer, W. Hollik and G. Weiglein, arXiv:hep-ph/0212037.

[16] K. Abe et al. [Belle Collaboration], Phys. Lett. B 511 (2001) 151 [arXiv:hep-ex/0103042];

S. Chen et al. [Cleo Collaboration], Phys. Rev. Lett. 87 (2001) 251807

[arXiv:hep-ex/0108032];

E. H. Thorndike [Cleo Collaboration], arXiv:hep-ex/0206067.

[17] A. Bartl, T. Kernreiter and W. Porod, Phys. Lett. B 538 (2002) 59 [arXiv:hep-ph/0202198].

[18] M. Carena, S. Mrenna and C. E. Wagner, Phys. Rev. D 60 (1999) 075010 [arXiv:hep-ph/9808312];

M. Carena, D. Garcia, U. Nierste and C. E. Wagner, Nucl. Phys. B 577 (2000) 88 [arXiv:hep-ph/9912516]; 
H. E. Logan, Nucl. Phys. Proc. Suppl. 101 (2001) 279 [arXiv:hep-ph/0102029];

H. Baer, J. Ferrandis, K. Melnikov and X. Tata, Phys. Rev. D 66 (2002) 074007 [arXiv:hep-ph/0207126];

C. Pallis, arXiv:hep-ph/0304047;

S. Profumo, Phys. Rev. D 68 (2003) 015006 [arXiv:hep-ph/0304071].

[19] J. P. Derendinger and C. A. Savoy, Nucl. Phys. B 237 (1984) 307;

J. A. Casas and S. Dimopoulos, Phys. Lett. B 387 (1996) 107 [arXiv:hep-ph/9606237].

[20] G. Sguazzoni, talk at the 31st International Conference on High Energy Physics, 25-31 July, 2002, Amsterdam; P. Lutz, talk at the same conference.

[21] M. Drees and K. Hagiwara, Phys. Rev. D 42 (1990) 1709;

G. Altarelli, R. Barbieri and F. Caravaglios, Int. J. Mod. Phys. A 13 (1998) 1031 [arXiv:hep-ph/9712368];

S. K. Kang and J. D. Kim, Phys. Rev. D 62 (2000) 071901 [arXiv:hep-ph/0008073].

[22] S. Bertolini, F. Borzumati, A. Masiero and G. Ridolfi, Nucl. Phys. B 353 (1991) 591.

[23] A. L. Kagan and M. Neubert, Eur. Phys. J. C 7 (1999) 5 [arXiv:hep-ph/9805303].

[24] N. Oshimo, Mod. Phys. Lett. A 4, 145, 1989.

[25] C. J. Damerell and D. J. Jackson, Prepared for 1996 DPF / DPB Summer Study on New Directions for High-Energy Physics (Snowmass 96), Snowmass, Colorado, 25 Jun - 12 Jul 1996;

K. Abe et al. [SLD Collaboration], Phys. Rev. Lett. 88, 151801 (2002)

[arXiv:hep-ex/0111035];

J. Abdallah [DELPHI Collaboration], arXiv:hep-ex/0311003.

S. M. Xella-Hansen et al., Linear Collider Note LC-PHSM-2003-061.

[26] S. Y. Choi, M. Drees, B. Gaissmaier and J. Song, arXiv:hep-ph/0310284. 